\title{
Imaging of Magnetic Textures in Polycrystalline FeGe Thin Films via in-situ Lorentz Transmission Electron Microscopy
}

Nuria Bagues ${ }^{1}$, Binbin Wang ${ }^{2}$, Tao Liu ${ }^{1}$, Camelia Selcu ${ }^{1}$, Stephen Boona ${ }^{1}$, Roland Kawakami ${ }^{1}$, Mohit Randeria ${ }^{1}$ and David McComb ${ }^{3}$

${ }^{1}$ The Ohio State University, Columbus, Ohio, United States, ${ }^{2}$ Center for Electron Microscopy and Analysis, The Ohio State University, Columbus, Ohio, United States, ${ }^{3}$ Center for Electron Microscopy and Analysis/ Department of Material Science and Engineering, The Ohio State University, Columbus, Ohio, United States

Recent years have seen enormous growth in research into the topologically stable spin textures in magnetic materials known as skyrmions. One major factor driving this growth is the potential application of skyrmions in magnetic data storage devices [1]. Developing methods for visualizing and characterizing these magnetic textures is a critical step in understanding their properties. This can generally be accomplished via Lorentz transmission electron microscopy (TEM), as demonstrated in single crystals and thin films of B20 FeGe [2,3,4]. Analysis of thin film samples is often complicated due to the microstructure resulting from the strain and growth process of the films. Grains, for example, contribute to image contrast in a way that can be difficult to separate from magnetic texture contrast, especially when the grains and skyrmions are similar in size. In this work, we report the successful application of an image subtraction method to reveal enhanced contrast of magnetic textures in an FeGe thin film, and discuss the potential for further enhancement of this contrast through Lorentz 4D scanning (S)TEM.

Here we report the use Lorentz TEM imaging to study magnetic textures in a polycrystalline FeGe thin film in plan view specimens. FeGe is a chiral magnet with a B20 structure and a helical transition at a Curie temperature of $T_{\mathrm{c}} \sim 280 \mathrm{~K}$ [2]. In this work, a 35nm FeGe film was grown on a Si[111] substrate via molecular beam epitaxy (MBE). High angle annular dark field (HAADF) STEM analysis was performed to characterize the structure and quality of the films in cross-section specimens prepared using focused ion beam (FIB). Plan view specimens were also prepared using wedge-mechanical polishing in order to image the magnetic textures. Lorentz TEM images were acquired at the same area under different conditions of temperature $(\mathrm{T})$ and field in order to emphasize either the magnetic contrast or grain contrast in the image. The grain contrast was then subtracted to more clearly observe the magnetic texture. In addition, energy dispersive x-ray (EDS) was performed to confirm the presence of the Si substrate in plan view specimens, and the FeGe grain orientation and size distribution was further studied via Transmission Kikuchi Diffraction (TKD) in a scanning electron microscope.

HAADF STEM imaging of the cross-section shows the FeGe thin film adopts a B20 structure and grows epitaxial on the Si substrate, following a [111] out-of plane direction, and [1-10] in-plane direction aligned with Si [11-2] (rotated $30^{\circ}$ with respect $\mathrm{Si}$ [1-10]), while plan view images clearly indicate the sample is polycrystalline. TKD results show ordered FeGe grains, with strong preference for growth along the [111] out-of-plane direction, as well as a tendency toward specific in-plane orientations rotated $60^{\circ}$ relative to each other.

Figure 1a shows a $200 \mu \mathrm{m}$ under-focused image acquired at room temperature and 0Oe field. Since this temperature is above $T_{\mathrm{c}} \sim 280 \mathrm{~K}$, no magnetic texture is expected under these conditions, and therefore all contrast in the image should come from sample defects such as grain boundaries. Figures $1 \mathrm{~b}$ and $1 \mathrm{c}$ correspond to images acquired at $240 \mathrm{~K}$ (below $T_{\mathrm{c}}$ ) in applied magnetic fields of $0 \mathrm{Oe}$ and $1300 \mathrm{Oe}$, 
respectively, which were chosen to cross between the regions of the magnetic phase diagram where we expect to observe only the general helical phase (0Oe) or the presence of skyrmions (1300Oe) [2]. To emphasize the magnetic contrast in these images, the features in figure 1a were subtracted from those in figure $1 \mathrm{~b}$ and figure $1 \mathrm{c}$ to produce figures $2 \mathrm{a}$ and $2 \mathrm{~b}$, respectively. With the magnetic texture contrast enhanced in this way, we can clearly observe stripes in figure 2a indicative of a helical magnetic phase, as well as distinguish between skyrmion lattices with different chirality based on the bright or dark contrast of the skyrmion centers in figure $2 \mathrm{~b}$. We note that previous reports suggest this helical phase is observed only if the substrate is completely removed [5], but in this study, EDS analysis confirms the Si substrate is still present.

We note that Lorentz 4D scanning (S)TEM has been proposed as another way to enhance magnetic contrast in polycrystalline materials [5]. After successfully applying the image subtraction method here in Lorentz TEM, we plan to compare these results with further analysis of the same sample via Lorentz 4D STEM, using a pixelated detector (EMPAD).

The results of this study demonstrate an effective image subtraction method for improving contrast of magnetic textures within polycrystalline plan view specimens, which we applied here to an FeGe film to identify the presence of a helical phase as well as the skyrmion lattices [6].
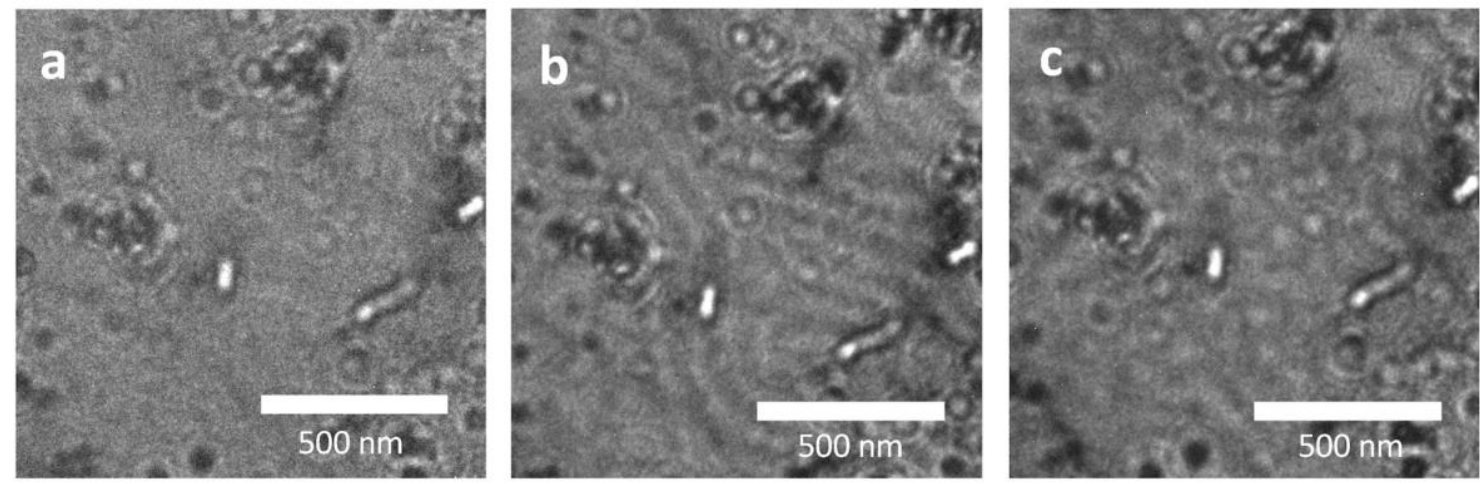

Figure 1. a), b) and c) correspond to Lorentz TEM images from the same area acquired with an underfocus of 200 microns at different conditions: a) room temperature and $0 \mathrm{Oe}, \mathrm{b}$ ) at $\mathrm{T}=240 \mathrm{~K}$ and $0 \mathrm{Oe}$, c) $\mathrm{T}=240 \mathrm{~K}$ and $1300 \mathrm{Oe}$.
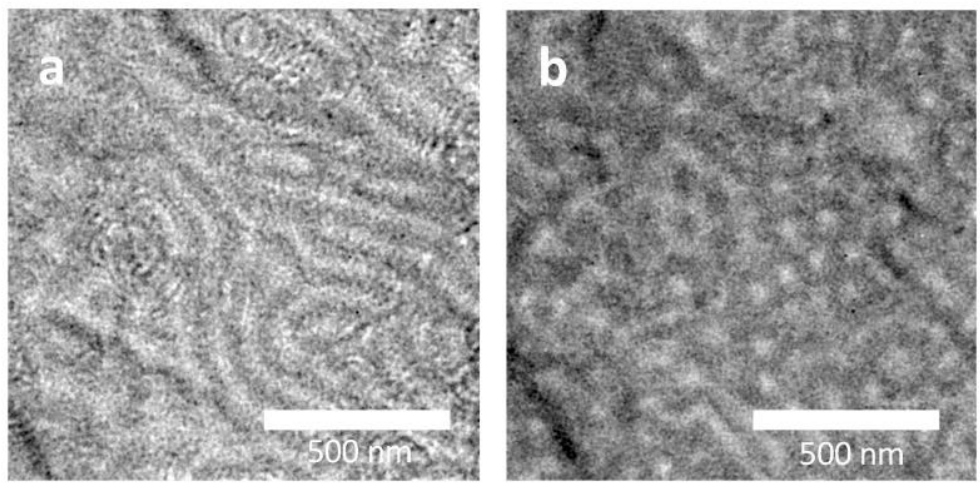

Figure 2. a) and b) correspond to the resulting images after subtracting figure 1 a to figure $1 \mathrm{~b}$ and to figure $1 \mathrm{c}$, respectively. These resulting images clearly show enhancement of the magnetic contrast, with the stripes in a) corresponding to the helical phase and the bright and dark central dots in b) comprising the skyrmion lattices of differing chirality. 


\section{References}

[1] N. Nagaosa and Y. Tokura, Nature Nanotechnology 8 (2013), p. 899-911.

[2] X. Z. Yu et al., Nature Materials 10, (2011), p 106-109.

[3] Z. A. Li et al., NanoLetters 17, (2017), p 1395 - 1401

[4] A. Adam et al., Physical Review Materials 2, (2018)

[5] K. X. Nguyen et al., arXiv:2001.06900 (2020)

[6] The authors acknowledge funding from Defense Advanced Research Projects Agency (DARPA) under Grant No. D18AP00008. 